\title{
Metaphors and problematic understanding in chronic care communication
}

\author{
Fabrizio Macagno*, Maria Grazia Rossi \\ ArgLab - Instituto de Filosofia da Nova (IFILNOVA), Universidade Nova de Lisboa, Edifício I\&D - 4 Andar, Avenida de Berna 26, $1069-061$ \\ Lisbon, Portugal
}

\section{A R T I C L E I N F O}

\section{Article history:}

Available online 5 April 2019

\section{Keywords:}

Misunderstanding

Interpretation

Pragmatics

Health communication

Intracultural communication

Metaphors

\begin{abstract}
A B S T R A C T
Metaphors can be used as crucial tools for reaching shared understanding, especially where an epistemic imbalance of knowledge is at stake. However, metaphors can also represent a risk in intercultural or cross-cultural interactions, namely in situations characterized by little or deficient common ground between interlocutors. In such cases, the use of metaphors can lead to misunderstandings and cause communicative breakdowns. The conditions defining when metaphors promote, and hinder understanding have not been analyzed in detail, especially in intracultural contexts. This study proposes an analysis of metaphors identified within an Italian corpus of diabetes care interviews. Through a coding scheme capturing the types and the probative weights of the linguistic evidence that can be used to detect misunderstandings, the communicative effectiveness of metaphors is indirectly assessed. The quantitative and qualitative analyses show a positive correlation between metaphor use and problematic understanding. A more detailed scrutiny of the interlocutors' roles and topics of the metaphors points out that most of the problematic metaphors are used by patients, while most of the problematic ones used by providers concern non-clinical matters. These results can be explained as resulting from incorrect presumptions of common ground between the interlocutors.
\end{abstract}

(c) 2019 Elsevier B.V. All rights reserved.

\section{Introduction}

In health communication, the importance of metaphors - and more importantly of the choice of metaphors used - has been stressed in several studies, which took into account different contexts of care and the distinct viewpoints of care providers and patients (Arroliga et al., 2002; Casarett et al., 2010; Demmen et al., 2015; Gibbs and Franks, 2002; Semino et al., 2015). Metaphors are regarded as tools for enhancing healthcare providers' ability to communicate effectively, emphatically, and clearly, and as strategies for patients to make sense and explain their illness. However, metaphors pose also a communicative challenge that results from the nature of metaphor understanding and the cultural differences in healthcare interactions.

Metaphor understanding has been shown to be essentially related to culture, or more precisely to sets of "conceptual mappings" that in many cases preexist communication, structuring "our thinking, reasoning, and understanding" (Gibbs, 1992, p. 596). The consequence of this relation is that metaphors can be interpreted correctly only if the interlocutors share the needed common ground. As Gibbs puts it (Gibbs, 1987, p. 574):

\footnotetext{
* Corresponding author.

E-mail addresses: fabrizio.macagno@fcsh.unl.pt, fabriziomacagno@hotmail.com (F. Macagno), mgrazia.rossi@gmail.com (M.G. Rossi).
} 
As with ambiguous language, the search for the acceptable interpretations of most metaphors will involve a large range of cultural conventions and mutually held beliefs, some of which may be quite idiosyncratic to particular people and contexts. [...] Thus, if two people use the word teapotted to mean something unique such as 'rubbing the back of someone's leg with a teapot', then the expression "John teapotted the policeman" can only by interpreted correctly by people who share this intimate knowledge.

Culture (in part correponding to the notion of common ground) at the same time shapes metaphors and guides their interpretation; however, the cultural dimension of metaphors and metaphor interpretation is also one of the potential causes of misunderstanding and miscommunication (Kovecses, 2015; Kövecses, 2005, 2010; Musolff, 2015). The impact of cultural differences on the interpretation of metaphors has been underscored in contexts of intercultural communication between native and non-native speakers, in which metaphor use was shown to cause comprehension difficulties (Littlemore et al., 2011; Musolff, 2014; Sharifian, 2014). The notion of intercultural communication, however, is only a way to refer to a degree of variation within the continuum of intra/inter-cultural communication (Kecskes, 2015). At any point of this continuum the common ground is not fully shared between interlocutors. This difference can be represented in terms of presumptions (Macagno, 2018a). While in intercultural communication the interlocutors cannot presume a large amount of information as part of the core common ground (the static, generalized, common repertoire of knowledge) (Kecskes and Zhang, 2013), in intracultural settings commonalities, conventions, standards and norms between speakers and hearers are usually taken for granted (Kecskes, 2013, 2015, 2016). Things might be more problematic when an epistemic imbalance is at stake, as in the case of medical communication. In such cases, problems of understanding may emerge due to major differences in terms of what interlocutors consider as part of their shared common ground (and culture). That is why metaphor understanding can be problematic also in intracultural contexts.

The purpose of this paper is to assess how the use of metaphorical expressions (Cameron, 2002; Casarett et al., 2010; Gibbs and Franks, 2002) affects understanding in a specific context of intracultural communication, namely Italian interviews between healthcare provider and patients in diabetes care. This type of interaction is characterized by the same linguistic code shared by the interlocutors, but also by crucial differences in their common ground resulting from the participants' knowledge (expert vs non-expert), age, social groups, and cultures (regional differences). To this purpose, we use a classification of the distinct types of evidence of misunderstanding (or, more broadly, problematic understanding) (Macagno, 2018b), defined as "mismatch between the speaker's intended meaning and the hearer's understanding of this meaning in the particular context of interaction" (Tzanne, 2000, p. 34). This classification is developed in a coding scheme for detecting problematic understanding according to both its nature and the strength of the evidence supporting its detection. This scheme will be used for analyzing a corpus of diabetes care interviews collected in Northern Italy, exploring the relationship between metaphors and problematic understanding, bringing to light the most frequent sources of potential problems of communication.

\section{Metaphors in intra-cultural communication: medical communication}

Metaphors are crucial instruments of communication. In particular, they are frequently used for explaining new or unclear concepts or highlighting some aspects of a concept that is then used to draw a further conclusion (Ervas et al., 2015; Macagno \& Zavatta, 2014). The different uses of metaphors and the communicative goals thereof need to be assessed considering the other dimension of metaphor use, namely its understanding. To this purpose, it is necessary to address the conditions that can affect metaphor understanding, and in particular the role of cultural differences.

According to the Aristotelian account, metaphors are defined as a type of "transference" based on logic-semantic relations (Aristotle, Poetics 1457b, 7-10), which can be carried out because there is no name for the concept indicated by the tenor (or in conceptual terms the target), or because there is a need to extend the meaning (or rather the associated commonplaces) of the tenor, which is achieved through the vehicle (Black, 1955, pp. 280-288). Metaphors are regarded as instruments that bring about a conceptual reorganization, extending the boundaries of a concept (Leech, 1981). For this reason, they are considered to have (among others) one fundamental purpose that is crucial in medical communication (Jaszczolt, 2002), namely making a concept or issue easier to understand (explanation).

The explanatory function of metaphors has been analyzed in contexts characterized by "epistemic imbalance," namely in which the interlocutors have different knowledge about a subject matter. In specialist-layman communication, metaphors have been described in terms of framing strategies that help understand something that is less familiar and less known in terms of something that is more familiar and already known (Schiappa, 2003; Semino et al., 2016). The similarities they create between the two domains of knowledge help understand some characteristics of the unfamiliar domain in terms of the more familiar one. For example, to explain why self-management is so important in the context of diabetes care, you can imagine describing the high levels of glycemia affecting people with diabetes in terms of pollutants into the blood that need to be kept under control. By framing the conceptual domain of diabetes in terms of the more familiar conceptual domain of pollution, some characteristics related to what the interlocutor already knows about pollution are used to explain the aspect of glycemia that matters most to the patient, namely the dangers and risks resulting from it. This dimension of metaphor has clearly a negative side, related to its selectivity. On the one hand, by highlighting some properties of the target, metaphors can hide others that can be relevant. On the other hand, metaphors create similarities that result in "emergent meanings not directly 
limited to speakers' or writers' communicative intentions" (Gibbs, 1992). For example, a patient can understand that glycemia is a substance that is only dangerous to the body, and that is external to it.

Metaphor understanding can be affected by the degrees in which information can be presumed as part of the common ground, and the difference between what is taken for granted and what is shared by the interlocutor. Our hypothesis is that this difference becomes problematic in the context in which speakers share the same language but have different cultural backgrounds - resulting from their belonging to different regions, social classes, or cultural or professional groups (Bigi, 2016; Rossi et al., 2017). This only apparently "purely" intracultural communication can lead to another source of problematic communication, deriving from the fact that speakers consider as common ground information that in fact defines a specific culture or subculture not linguistically different.

The context of healthcare communication is a clear case of communication across different cultures sharing the same linguistic code. Healthcare communication is an asymmetrical communicative contexts characterized by an epistemic imbalance between the patient and the healthcare provider (Bigi 2011, 2014a). A bi-asymmetrical distribution of knowledge and procedures define the communicative interactions in this context (Rossi, 2016). Healthcare providers have an epistemic advantage, as they have privileged or superior knowledge of procedures, therapeutic regimen, and clinical information. Patients have an epistemic advantage relative to information about their own subjective experience with illness. This type of information can be particularly helpful in establishing diagnoses, plays a key role in disease monitoring and deciding upon treatment options. One crucial goal of medical interviews is to bridge this epistemic gap to make a sound recommendation, namely not only prescribing drugs, but also agreeing upon a course of action that the patient needs to comply with in order to prevent complications (Bigi, 2014b; Epstein and Gramling, 2012; Epstein and Street, 2011; Street et al., 2009; Wagner et al., 2001). The sharing of both clinical (from the provider's side) and personal and value-related (from the patient's side) information is crucial for this purpose.

In this context, metaphors can be regarded as tools for enhancing healthcare providers' ability to communicate effectively, emphatically and clearly, and as strategies for patients to make sense and explain their illness (Demjén and Semino, 2016; Rossi, 2016). Metaphors can convey new information through familiar concepts related to the patients' background (Keysar and Glucksberg, 1992, p. 654). The use of metaphors can regulate how patient view and project their illness, and thus can be used for making shared decisions that can be understood and accepted (Gibbs and Franks, 2002). From the perspective of healthcare providers, metaphors can also be regarded as an important component of communication and linguistic competence (Bleakley, 2017; Crawford et al., 2017; Demjen et al., 2016; Semino et al., 2018), useful for both explaining medical information and improving the shared decision-making between patients and providers (Álvarez et al., 2017). Moreover, the use of metaphors in clinical encounters has been shown to lead to facilitating understanding, engaging patients and increasing their self-management abilities (Naik et al., 2011).

However, metaphors are not all equal and are not all used for the same purpose (Trogen, 2017; Ervas et al., 2016). Metaphors can be used differently for pursuing a wide range of goals including explaining, summarizing, supporting a viewpoint, illustrating, clarifying or persuading (Cameron, 2003; Goatly, 2011; Semino, 2008). Moreover, not all metaphors can be considered as equally effective from a communicative point of view, or more precisely, not all metaphors can lead to the "the smooth progression" of discourse that is a sign of understanding in discourse. Not only do some metaphors fail to avoid misunderstandings, but they can cause or result in the "turbulence" in discourse that is a sign of problematic understanding (Mauranen, 2006, p. 128).

Medical communication is a crucial context for analyzing metaphor understanding. On the one hand, metaphors are and need to be used in this context for pursuing different goals and most importantly explaining concepts to interlocutors unfamiliar with them. On the other hand, the epistemic unbalance between the interlocutors defines this type of communication and often combines with other cultural differences - resulting from belonging to different social, regional, age, and professional groups. These intracultural differences can lead to the risk that the interlocutor cannot access the information (or the system of related commonplaces, see Black, 1955, p. 288) needed for interpreting and reconstructing the metaphorical meaning. This cultural gap can thus result in the risk of misinterpreting or failing to interpret metaphors, thus affecting their communicative effectiveness. This study intends to advance some methodological tools to assess whether metaphors can lead to problematic understanding in diabetes care communication, and in what circumstances.

\section{Detecting metaphors}

To address the analysis of metaphor understanding in the specific intercultural context of medical communication, we designed a study based on a corpus of diabetes care interviews collected in Northern Italy (Bigi, 2014a). The aim was to assess the communicative effectiveness of the metaphors used by patients and healthcare providers from a linguistic point of view. To this purpose, we detected metaphors and used a classification of distinct types of linguistic evidence of problematic understanding (Macagno, 2018b) to bring to light which metaphors were between the most frequent sources of potential communicative problems.

Based on the leading studies conducted in the field of applied linguistics and metaphor studies, metaphor understanding was analyzed by considering exclusively the linguistic level (Cameron and Maslen, 2010; Gibbs, 1994), focusing on the linguistic evidence that can constitute a significant but only indirect proof of metaphorical understanding. A linguistic assessment of metaphors is not enough to determine the understandability of metaphors per se, in terms of the processes and 
inferences behind their psychological interpretation. However, it can provide a sign that their effectiveness in terms of understanding is problematic (Macagno, 2018b).

\subsection{Corpus description}

We analyzed a corpus of interviews in Italian between diabetes patients and healthcare providers, recorded from March 2012 through March 2014 in the diabetes outpatient clinic of the A.O. I.C.P. - Cusano Milanino, Milan, Italy (total number of visits $N=60$ ). The Ethical Committee of the I.C.P approved the protocol in January 2012. Written informed consent was obtained from all participants (Bigi, 2014a). The detection of metaphors and misunderstandings has been conducted on 46 consultations involving 13 patients $(\mathrm{N}=7$ females) and 6 healthcare providers $(\mathrm{N}=6$ females) (Table 1$)$. The interviews selected for coding were chosen according to 1 ) quality of the recording (interrupted or corrupted videos were excluded $\mathrm{N}=6$ ); 2) duration and goal of the consultation (extremely short visits involving only medical report checking were excluded $-\mathrm{N}=8$ ).

Table 1

Patients and healthcare providers characteristics.

\begin{tabular}{ll}
\hline Patients $(\mathbf{N}=\mathbf{1 3})$ & \\
Female & $\mathrm{N}=7$ \\
Southern Italy & $\mathrm{N}=9$ \\
Total number of visits with female & $\mathrm{N}=25$ \\
Education: high school & $\mathrm{N}=2$ \\
Education: middle school & $\mathrm{N}=3$ \\
Education: primary school & $\mathrm{N}=8$ \\
Healthcare providers $(\mathbf{N}=\mathbf{6})$ & \\
Female & $\mathrm{N}=6$ \\
Physician & $\mathrm{N}=3$ \\
Nurse & $\mathrm{N}=2$ \\
Dietician & $\mathrm{N}=1$ \\
\hline
\end{tabular}

The vast majority of the patients have a level of formal education that does not go beyond primary school (almost 70\%), while only $15 \%$ of them holds a high school diploma. Considering the regional provenance, which in Italy defines also dialects, the difference between providers and patients is noticeable. While most of the patients are from the South $(\mathrm{N}=8)$, all the providers are from the North (and in particular from Lombardia, a northern region). On average, the patients were diagnosed with diabetes 115 months before the recorded visit at the health center $(S D=76.2)$. The number of recording per participant varies both relative to the patients (mean 3,6; range 2-6) and healthcare providers (mean 7.7; range 1-12). Overall, we looked at $23(50 \%)$ interviews with nurses, 18 (39\%) interviews with physicians and $5(11 \%)$ interviews with the dietician. In some consultations $(\mathrm{N}=11 ; 24 \%)$, patients were accompanied by family members $(\mathrm{N}=5)$; daughters $(\mathrm{N}=2)$; wives $(\mathrm{N}=2)$, husband). However, the percentage of their turn $(\mathrm{N}=886)$ represents only $4.7 \%$ of the total $(\mathrm{N}=18,679)$ and $9,6 \%$ of the patients' turns $(\mathrm{N}=9207)$. In what follows, we provide further specification of our methodological approach.

\subsection{Coding metaphors}

The metaphors used by patients and healthcare providers in our corpus of medical interviews have been manually identified (Black, 1979; Cameron, 1999; Semino, 2008; Wikberg, 2004). The assumptions underlying our coding were the following: 1) the principle that "the rules of our language determine that some expressions must count as metaphors" (Black, 1955); and 2) the focus on the metaphorical comparison, and not strictly on the expression thereof (Steen, 2010), which resulted in the inclusion of the so-called "direct metaphors" (including similes). The procedure for metaphor identification was developed starting from the MIPVU guidelines (Metaphor Identification Procedure VU University Amsterdam) (Steen et al., 2010), which was modified and adapted to the specific issues characterizing the corpus. The most important steps that we followed are indicated below:

1. The coders - familiar with both the subject matter and the type of communication - read the whole corpus and then each transcribed visit without performing any coding. This step is necessary for familiarizing with the lexical choices of the doctors and patients.

2. The coders take into account the lexical units. For each unit:

a. Establish its contextual meaning;

b. Determine whether the contextual meaning is different from the "literal" meaning as perceived by the coder as a native speaker;

c. Determine whether there is an incongruity between Topic and Vehicle domains. 
3. In case the contextual meaning is perceived as different from the "literal" one, the dictionary definition of the lexical unit is determined by consulting the Italian dictionary Treccani.

4. If the contextual meaning contrasts with the "literal" (or basic) meaning, but it can be understood in comparison with it, the unit is marked as metaphorical.

These steps were considered only as general guidelines, as the "boundary between literal and metaphorical language" is often fuzzy (such as in case of lexicalized metaphors, idioms, delexicalized words) (Semino et al., 2004, p. 1277). We needed to consider a contextual perspective, namely what can potentially count as a metaphor for the interlocutors in our corpus. Two problems arise in a communicative setting characterized by a cultural difference between the interlocutors (in our corpus, resulting from both educational and regional differences). The first problem is at the level of the step 2c, as the detection of an incongruity depends on contextual and background knowledge (Cameron, 1999). In our corpus, providers use expressions (such as "profile" to indicate a series of glycemia tests that need to be done before and after a meal) that are idiomatic in a specific chronic care community, but that cannot be presumed as such by individuals that do not belong to it, or who have only recently accessed it. In this case, we analyzed the common context of use of a term, relying on the corpus linguistics software Sketch Engine.7 (Kilgarriff et al., 2014), which provides a standard Italian corpus (Italian Web 2016). If the contextual meaning of the lexical item in our corpus does not correspond to the lexical one nor to the common contextual meaning, we presume that it is an instance of potential non-idiomaticity. For example, "profile" is commonly used to refer to the outline of physical entities (buildings, etc.), but not to a scheme of measurement. For this reason, we cannot presume that in the given context the term is perceived as idiomatic, and we code it as a metaphor. The second problem is the use of delexicalized verbs, such as "mettere (su peso)" (to put on weight) or "perdere (peso)" (to lose weight), which are the most frequent in our corpus. In this case, we follow the criterion of establishing the primary meanings of such words, and the conditions for their non-metaphorical use (Cameron, 1999). For example, "to put" has as a primary meaning in Italian "to place something in a specific position," while "to lose" is commonly understood as "to be unable to find something," which leads to considering as metaphorical the phrases "to put (a characteristic of the body) (on the body)" or "to lose (a characteristic of the body)." A more complex case concerns the different uses of "andare" (to go). The use of "to go" to mean "how are you?" ("come va?") is coded as non-metaphorical, as the meaning of "developing" or "occurring" is considered as a primary meaning of the verb and can be attributed to the implicit subject (life, existence...). In contrast, the expression "dove andava (the diabetes)" ("where the diabetes was going") means a physical movement and presupposes a physical entity. For this reason, we code this use of the verb as metaphorically attributed to a condition (the illness).

In borderline or uncertain cases, the criteria mentioned above were combined with an additional criterion, consisting in determining whether the alleged metaphors can have a "literal" interpretation. In case it is possible to interpret the allegedly metaphorical expression non-metaphorically, then it can be presumed to be metaphorical. For example, while in Italian it is possible to interpret "losing weight" and "putting on weight" to refer to uploading or unloading weight, the meaning of "come va?" cannot be interpreted in other ways. The cases of doubt were discussed and resolved between coders, considering the dimensions of idiomaticity and novelty of a potential metaphor for the interlocutors in the specific corpus.

\section{Coding problematic understanding}

To analyze actual or potential problems of communication we developed a coding scheme based on a classification of linguistic evidence of problematic understanding, which we can define as a potential mismatch between speaker's intended meaning and the hearer's understanding thereof, or (negative definition) as a case in which the hearer's understanding cannot be presumed. The instances of problematic understanding ${ }^{1}$ can be detected in texts based on linguistic clues, namely types of evidence having different probative weights (Walton, 2016). The evidence of problematic understanding was classified in three categories, namely strong $(S)$, acceptable $(A)$, and weak $(W)$. (S) corresponds to direct meta-dialogical evidence, (A) to indirect meta-dialogical evidence, and (W) to indirect dialogical evidence (see Fig. 1 below).

The distinction between direct and indirect evidence concerns the communicative effects of misunderstandings. The direct effects are cognitive and consist in the hearer's reaching an interpretation that contradicts his background assumptions. Such effects, called puzzled understanding (Yus, 1999), are made manifest by meta-dialogical requests of clarification or explanation. Indirect effects include the communication failures, breakdowns, and communicative problems that may result from misunderstandings that have not been clarified or other causes. The distinction between dialogical and meta-dialogical evidence (Macagno, 2018c) reflects the dialogical nature of understanding, in which the construction of next turns in a dialogical interaction depends on the understanding of the prior turns (Sacks et al., 1974), or the repair of the misunderstanding thereof (Schegloff, 1987; Schegloff et al., 1977). In the first case, the speaker engages in the dialog by continuing it (either relevantly or irrelevantly). In the second case, one of the interlocutors suspends or interrupts the dialog (Gumperz and Tannen, 1979) and discusses, requests, or verifies the interpretation of an utterance.

\footnotetext{
${ }^{1}$ We will use the term "misunderstanding" to refer to the thus defined concept of "instance of problematic understanding."
} 
Table 2

Coding categories described.

\begin{tabular}{|c|c|c|c|c|}
\hline Name & Code & Description & Example & $\begin{array}{l}\text { Prob. } \\
\text { weight }\end{array}$ \\
\hline $\begin{array}{l}\text { 1) Declaration of lack of } \\
\text { understanding }\end{array}$ & LACK & $\begin{array}{l}\text { The hearer acknowledges meta-dialogically that } \\
\text { s/he cannot understand the whole move, or that } \\
\text { the interpretation that s/he has achieved is not } \\
\text { acceptable. It can be caused by the impossibility } \\
\text { of decoding part of the sentence, or by a conflict } \\
\text { between the hearer's interpretation and other } \\
\text { stronger background assumptions. }\end{array}$ & $\begin{array}{l}\text { Patient: then I noticed that if I eat gnocchi, it }<\text { the } \\
\text { glycated hemoglobin }>\text { empties itself quickly. I } \\
\text { love gnocchi so much } \\
\text { Nurse: How does it empty itself quickly??? } \\
\text { Patient: eh it goes down, goes down }\end{array}$ & Strong \\
\hline $\begin{array}{l}\text { 2a) Alternative } \\
\text { interpretation of the } \\
\text { semantic } \\
\text { representation } \\
\text { ("semantic" } \\
\text { interpretation) }\end{array}$ & SEM ALT & $\begin{array}{l}\text { The hearer interprets the speaker's move by } \\
\text { specifying its meaning in a way that is not } \\
\text { acceptable or accepted. The speaker corrects the } \\
\text { hearer's alternative interpretation of the } \\
\text { semantic representation of his/her utterance. The } \\
\text { semantic representation refers to the } \\
\text { phenomena referred to as "explicatures" in } \\
\text { addition to syntactic or semantic disambiguation. }\end{array}$ & $\begin{array}{l}\text { Doctor: But I need you to make plan it in a more } \\
\text { reasoned manner. } \\
\text { Patient: Every day? } \\
\text { Doctor: We can also split it, you don't need to } \\
\text { plan it all in a day. But do it as a pair. I mean that } \\
\text { you have to do them before a meal and after the } \\
\text { meal }\end{array}$ & Strong \\
\hline $\begin{array}{l}\text { 2b) Alternative } \\
\text { interpretation of the } \\
\text { intended purpose of } \\
\text { speaker's utterance } \\
\text { ("pragmatic" } \\
\text { interpretation) }\end{array}$ & PRAG ALT & $\begin{array}{l}\text { The hearer interprets the speaker's move by } \\
\text { drawing inferences that are not acceptable or } \\
\text { accepted. The speaker corrects the hearer's } \\
\text { alternative interpretation of the intended } \\
\text { purpose of speaker's utterance. The intended } \\
\text { purpose includes implicatures, presuppositions, } \\
\text { or in general pragmatic inferences drawn from } \\
\text { an utterance. }\end{array}$ & $\begin{array}{l}\text { Doctor: Due to this, now it is hard for me to say } \\
\text { whether you should or should not take the evening } \\
\text { pills, as there are some evenings in which... } \\
\text { Patient: No no, you do not have to remove these } \\
\text { pills from my health-care program, they are not } \\
\text { bothering me. } \\
\text { Doctor: No, this is not the point. The point is that } \\
\text { there are evenings in which you eat a bit too much, } \\
\text { and others in which you do not eat at all. }\end{array}$ & Strong \\
\hline 3) Clarification request & CLA & $\begin{array}{l}\text { The hearer asks the speaker to specify better the } \\
\text { meaning of his utterance, as some of its } \\
\text { component can have different interpretations. } \\
\text { No interpretative hypothesis is advanced; only a } \\
\text { question is asked concerning a component of the } \\
\text { speaker's utterance. }\end{array}$ & $\begin{array}{l}\text { Wife: Can he eat fruit? Because he eats quite a lot } \\
\text { of fruit, eh. } \\
\text { Doctor: What? What does "quite a lot" mean? } \\
\text { Wife: Fruit, orange... } \\
\text { Doctor: No, what does "QUITE A LOT" mean? } \\
\text { Patient: An orange after a meal - or an apple. } \\
\text { Doctor: Then it is important that you eat only one } \\
\text { fruit after a meal. }\end{array}$ & Acceptable \\
\hline $\begin{array}{l}\text { 4) Check of } \\
\text { understanding }\end{array}$ & CHECK & $\begin{array}{l}\text { The hearer expresses a doubt of understanding, } \\
\text { as s/he is uncertain to have understood correctly } \\
\text { what the speaker said. }\end{array}$ & $\begin{array}{l}\text { Patient: I skipped some checks with the device for } \\
\text { the blood glucose monitoring. } \\
\text { Doctor: You did less profiles. } \\
\text { Patient: Less. Yes, yes. }\end{array}$ & Acceptable \\
\hline 5) Irrelevance & IRR & $\begin{array}{l}\text { The hearer advances a move that is incoherent } \\
\text { either pragmatically or topically (change of } \\
\text { subject) with the speaker's one. Pragmatic } \\
\text { incoherence can be for example a request of } \\
\text { information followed by an acknowledgment, or } \\
\text { the repetition of the speaker's previous utterance } \\
\text { after the hearer's turn. }\end{array}$ & $\begin{array}{l}\text { Doctor: It is too much. Well, you have got to do } \\
\text { something. That's your job, not mine. } \\
\text { Patient: I am having an ultrasound scanning } \\
\text { tomorrow. }\end{array}$ & Weak \\
\hline 6) No uptake & NO UP & $\begin{array}{l}\text { The hearer fails to take into account the other's } \\
\text { move by interrupting the dialog (silence) or } \\
\text { continuing the dialog without considering the } \\
\text { interlocutor's turn. }\end{array}$ & $\begin{array}{l}\text { Context: The nurse is running a foot examination; } \\
\text { she has already recommended using more skin } \\
\text { cream products. } \\
\text { Patient: My wife underwent a surgery. } \\
\text { Nurse: Look at that dry, dry skin. }\end{array}$ & Weak \\
\hline 7) Anticipation & ANT & $\begin{array}{l}\text { Clarification of an utterance provided directly by } \\
\text { the speaker, without being requested by the } \\
\text { hearer. }\end{array}$ & $\begin{array}{l}\text { Doctor: For example, another comfortable fruit is } \\
\text { the strawberry. } \\
\text { Doctor: because you do not need to peel it. }\end{array}$ & \\
\hline
\end{tabular}

The speaker can thus assess whether his or her utterance has been successfully understood (providing additional clarification if needed), or the hearer expresses his or her doubtful or otherwise problematic understanding. Meta-dialogical evidence of misunderstanding can be either direct (S) or indirect (A) evidence of problematic understanding (Clark and Brennan, 1991).

This theoretical distinction led to the coding categories that are represented in the following Table 2:

These types of evidence can be summarized in Fig. 1, which distinguishes the types of evidence according to their strength (Strong, Acceptable, Weak) and the type of problematic understanding they reveal (lack of understanding: 1, plus 5 and 6; alternative understanding: $2 \mathrm{a}$ and $2 \mathrm{~b}$, plus 5 and 6 ; doubtful understanding: 3; non-presumable understanding: 4). 


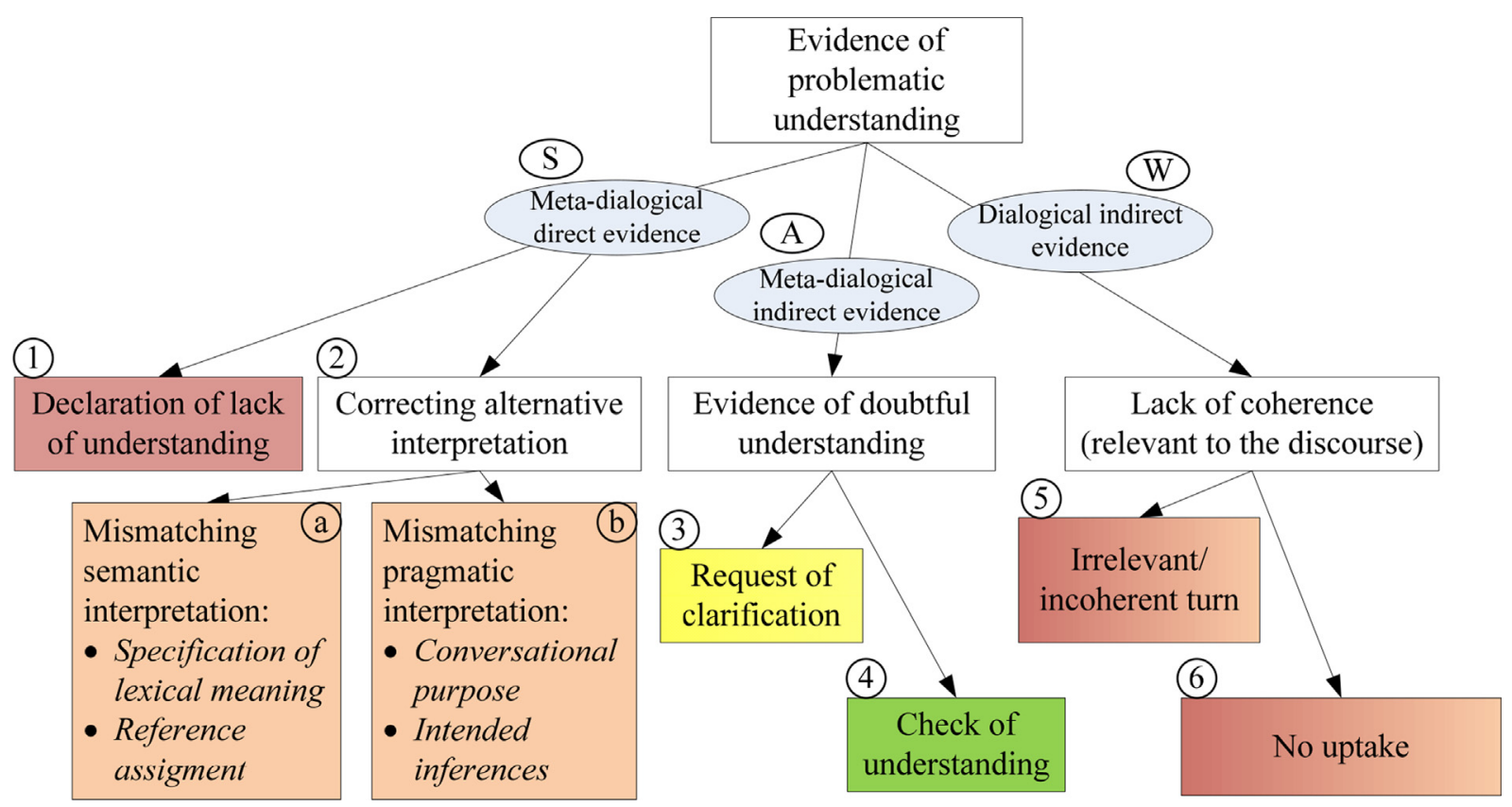

Fig. 1. Evidence of problematic understanding.

This classification allows the analyst to detect when understanding can be problematic, namely when the speaker cannot or does not presume that the interlocutor has understood or will understand what has been said, and why. The force of the evidence can be used to justify the occurrence of problematic understanding, namely identifying when this conclusion can be grounded on strong, plausible (acceptable), or weak evidence. Clearly, different types of evidence can combine and result in higher probative weight (Walton and Reed, 2008). For example, irrelevance (5) (such as a turn incoherent with the pragmatic purpose of the previous turn) can be followed by a request of clarification (3), or a repetition of the previous turn (5). The combined pieces of evidence support more strongly the conclusion that a misunderstanding has occurred than the evidence individually considered. The types of problematic understanding detect whether the mismatch of interpretations actually occurred (lack of understanding; alternative understanding; irrelevance; no uptake) or could have occurred (request of clarification; check of understanding).

To these dialogical cues of problematic understanding, evidence of non-presumability of an interpretation can be added, detecting cases in which the speaker is aware that his/her utterance is potentially problematic. The anticipation of problematic understanding (ANT) captures the clarification of an utterance provided directly by the speaker. This type of nonrequested clarification shows that the utterance cannot be taken for granted as (univocally) understandable by the hearer, as a specification of its meaning is provided in advance.

\section{Metaphors and misunderstandings in patient-provider communication}

Together with the category of anticipation (ANT), we have used the seven categories described in Fig. 1 (LACK, SEM ALT, PRAG ALT, CLA, CHECK, IRR, NO-UP) to codify linguistic evidence of problematic understanding in the corpus of medical interviews we described above. The interrater reliability was calculated by independent coders on $35 \%$ of the corpus and was strong with 99,1\% agreement and a Krippendorff's Alpha $=0.89$. The interrater reliability for the 7 more specific categories of problematic understanding and the ANT category is reported in the table below (Table 3):

Table 3

Interrater reliability for the 7 categories of problematic understanding and anticipation.

\begin{tabular}{lll}
\hline Category & Agreement & Krippendorff's alpha \\
\hline LACK & $99,7 \%$ & 0,946 \\
SEM ALT & $98,5 \%$ & 0,876 \\
PRAG ALT & $96,4 \%$ & 0,865 \\
CLA & $96 \%$ & 0,863 \\
CHECK & $93 \%$ & 0,768 \\
IRR & $99,1 \%$ & 0,875 \\
NO-UP & $100 \%$ & 1 \\
ANT & $95,7 \%$ & 0.88 \\
\hline
\end{tabular}


The first broad category in our classification system is defined as "meta-dialogical direct evidence," and includes three categories: lack of understanding, semantic alternative understanding, and pragmatic alternative understanding. A clear case is the following, in which the nurse reaches an interpretation that is not acceptable and declares her lack of understanding of the phrase "to empty itself" (characters in bold identify the metaphors; the underlined text refers to the problematic understanding).

Case 1: Lack of understanding

1. Paziente: Poi ho notato che se mangio gli gnocchi, mi si svuota in fretta. A me piacciono tantissimo.

2. Infermiera: Come si svuota in fretta?

3. Paziente: Eh va giù, va giù.

1. Patient: Then I have noticed that if I eat gnocchi, it empties itself quickly. I love gnocchi very much.

2. Nurse How does it empty itself quickly?

3. Patient: $\quad$ Eh it goes down, goes down.

The nurse's reply is meta-dialogical in the sense that she declares her impossibility to interpret part of the patient's utterance (2). The nurse can decode the utterance, but cannot accept or enrich the semantic representation obtained, as it conflicts with her background assumptions (to her knowledge, nothing related to diabetes can be filled in or emptied).

The second type of meta-dialogical evidence of misunderstanding consists in the correction of an alternative interpretation, which can be at the level of the enrichment of the semantic representation or the pragmatic inferences that can be drawn from it (we can consider this distinction as corresponding to the notion of explicit vs implicit dichotomy in Relevance Theory, see (Carston, 2009). We use the terms "semantic" vs. "pragmatic" alternative understanding to refer to two distinct type of mismatches between the interlocutors' interpretations, both resulting from pragmatic processing (Sperber and Wilson, 1995). An example of an alternative interpretation of the semantic representation is the following case, in which the physician is talking about the glycemic controls to a patient already accustomed with this practice - consisting in random controls before and after the meals.

\section{Case 2: Alternative interpretation of the semantic representation}

1. Dottoressa: Però mi servirebbe mh per capire anche meglio, farlo un pochino più ragionato.

2. Patient: Tutti i giorni?

3. Dottoressa: Ma anche possiamo anche dividerlo, non farlo tutto il giorno, però a coppia, nel senso, Io tipo dovrei farli mh, prima del pasto e dopo il pasto.

1. Doctor: But I need $\mathrm{mh}$ also to better understand, you to make it in a little more reasoned manner.

2. Patient: Every day?

3. Doctor: But also we can also split it, you don't need to do it all in a day, but do it in pairs, in the sense that you should do them before a meal and $\mathrm{mh}$, after a meal.

The physician uses the phrase "in a more reasoned manner" to refer to the modality the controls need to be made (coupled controls, before and after eating). His own explicature of the utterance is "You should plan the control in a more reasoned $<$ from the point of view of the modality $>$ manner." However, the physician's explicature conflicts with the patient's, who interprets the phrase $<$ from the point of view of the frequency $>$ (2). The physician corrects the alternative explicature of the patient by specifying explicitly the intended meaning (3) (Capone, 2009; Macagno \& Capone, 2016).

The correction of an alternative understanding can be also focused on the pragmatic inferences that can be drawn from an utterance, which can be related to the information conveyed or the communicative purpose pursued (Leech, 1983, p. 99). An example of the evidence of a mismatch between the inferences that can be drawn from an utterance is the following:

Case 3: Alternative pragmatic inferences

1. Infermiera: A maggio, sì non ci s- lei ha scade quindi deve prendere del materiale?

2. Paziente: Devo fare il piano terapeutico per andare a rifare tutto il materiale.

3. Infermiera: Ma materiale a casa ne ha? Ha recuperato l'ultima manche di materiale, com'è messa?

4. Paziente: Devo ritirare le strisce ad aprile.

1. Nurse: In May, yes. I will not she has it expires, so do you need to fetch some material?

2. Patient: I need to have the therapeutic plan in order to redo the whole material.

3. Nurse: But do you have material at home? Have you withdrawn the last parcel of material, how is the situation?

4. Patient: I have to withdraw the strips in May.

The nurse interprets the patient's utterance as implying that she needs to have a new therapeutic plan to have the whole material needed for self-controlling the levels of glycemia. For this reason, she concludes that he has no material and cannot get it until the new plan is issued (3). The patient, however, replies correcting the inference (4, she can get the material in April). This mismatch of pragmatic interpretations stems from the explicature of the metaphor "to redo (the material)," which the nurse interprets are "to restock (the provisions of material)" and the patient as "renew the possibility of withdrawing the material." 
The correction of an alternative pragmatic interpretation can be also at the level of the purpose of the utterance. An example is Case 2 above. While the evidence available identifies directly only a different explicature of the utterance, it is possible to notice that this alternative understanding is a sign of a pragmatic misunderstanding. While the doctor intended to complain about the incorrect self-management of the patient, the latter instead interprets the utterance as a suggestion of modifying the control frequency.

Meta-dialogical evidence of a possible problematic understanding can be indirect, namely cues that can be explained as caused by problematic understanding. Clarification requests can be considered as evidence that the interlocutor's utterance can be problematic because not sufficiently specific. For this reason, the hearer asks the speaker to specify better the meaning of his utterance, as it can have different interpretations. A clear case is provided by the following excerpt, in which the specific meaning of "to let oneself go" is unspecific.

Case 4: Clarification request

1. Infermiera: mi dice che si è lasciata andare

2. Paziente: eh sì

3. Infermiera: cosa succede?

4. Paziente: eh visto che gli esami erano buoni insomma

5. Infermiera: ha modificato qualcosa nello stile di vita, per dire un po' di-

6. Paziente: ho modificato con qualche dolce in più, onestamente.

1. Nurse: You're telling me that you've let yourself go

2. Patient: Yes.

3. Nurse: What happens?

4. Patient: [eh considering] that the tests were going well..

5. Nurse: You have changed something in your lifestyle, a bit more of-

6. Patient: I've changed it with some desserts more, honestly.

The nurse asks a more specific description (3) of the action indicated through the metaphor (1). The patient provides an explanation (4), which the nurse completes and proposes as a possible interpretation for confirmation (5). As a result, the patient clarifies the exact meaning of his utterance in the last turn (6).

The nurse's last turn in Case 4 provide indirect evidence that the understanding of the utterance cannot be presumed to be doubtless. For this reason, the hearer checks whether he or she has understood correctly, leaving the possibility to the speaker to correct or specify further the interpretation. The check of understanding is a strategy used for ascertaining the correctness of the reconstruction of the semantic representation or the intended pragmatic inferences in case of doubt. A clear example is the following.

\section{Case 5: Check of understanding}

1. Paziente: mi sembra di sì. cioè, le dirò che questo periodo sono un po' sballata un po' anche di testa diciamo non sono:: regolare::

quindi ho saltato:: di scri- no eh:: le pastiglie sempre:: ho saltato dei: controlli:: con la:: macchinetta ecco

2. Dottore: si è fatta meno profili

3. Paziente: meno si si

1. Patient: I think yes. Or rather, I tell you that in this period I am a bit messed up, also at the level of my head I am not regular. Therefore

I have jumped the writing ... no the pills always ... I have jumped some controls with the machine.

2. Doctor: You have done less profiles

3. Patient: Less, yes yes

The nurse checks the explicature of "jumping some controls," and more precisely the interpretation of the metaphor as "failing/forgetting to do some profiles" (and not, for example, postponing them). The patient then confirms the explicature, which is clear evidence of a check of understanding.

The last category of evidence is dialogical indirect evidence. A clear case is provided by the following excerpt, in which the patient complains about the effects of the cortisone $(2 ; 4)$ which she describes as causing her "swelling." The nurse, however, interprets the verb (to swell) as "to increase in weight," not "to dilate," and continues to praise the patient for her positive selfmanagement $(3 ; 5)$, without addressing the patient's expression of a preoccupation (7).

\section{Case 6: Irrelevance and lack of uptake}

1. Infermiera: Però di base lei deve stare attento. Non si è alzato molto con il peso, direi. Pensavo peggio. Invece no, è stato bravo.

2. Paziente: No perché gonfio col cortisone.

3. Infermiera: Peso stabile proprio. Il peso veramente stabile. Allora

4. Paziente: Sono gonfio per que- que- il cortisone, quello che mi fa gonfiare.

5. Infermiera: Esame del piede nella norma. quand'è che ha fatto l'operazione.

1. Nurse: However, basically you shall be careful. You have not gone up with your weight. I thought it was worse, but no, you have done well.

2. Patient:

3. Nurse:

4. Patient:

5. Nurse: No, I swell. Using the cortisone I swell.

Weight stable indeed. The weight is really stable. Then

I am swollen because of this.... The cortisone, that makes me swell.

The foot control is normal. When did you undergo the surgery? 
The misunderstanding is at the level of the specific semantic representation of the utterance. The nurse relies on the explicature <to grow in bulk due to physiological factors>, which leads to inferring that the patient is worried to being increased in weight. In contrast, the patient relies on the explicature <increase in volume due to abnormal factors $>$, which can be the most accessible one in a context in which the effects of drugs are discussed. The irrelevance is thus a sign of an alternative understanding (3). We underscore that this evidence of problematic understanding is only a possible sign of an actual misunderstanding. The patient may have expected a relevant move by the nurse and may have interpreted the irrelevance as a sign of misunderstanding of the metaphor "to swell," which led him to overinterpreting the metaphor. Also in this case, the irrelevance is interpreted by the patient as a sign of problematic understanding. The last turn is also characterized by a piece of indirect dialogical evidence of problematic understanding. The nurse fails to continue to dialog by addressing the patient's worries, and instead she shifts the discussion to a different and not related topic (5, foot control). This case can be considered as a lack of uptake (the reply is not related to previous turn) and can indicate the nurse's failure to understand patient's move as a request of help (related to the provision of worrisome information).

Case 6 shows how both irrelevance and lack of uptake can be only weaker signs of problematic understanding. Both the first and second instance of dialogical evidence can be explained not as signs of problematic understanding, but as refusals to engage in the conversation further due to time constraints (the nurse needs to continue the visit).

\section{Metaphors and understanding - a linguistic assessment}

The sections above have illustrated the theoretical framework who led the building of the coding scheme we have developed for the identification of the types of problematic understanding and the conclusiveness of the various types of evidence available. This coding scheme has been applied to the aforementioned corpus for investigating the associations and effects between metaphors and understanding. In this section we discuss our findings to offer a linguistic assessment of the metaphors used in the context of diabetes care by patients and healthcare providers.

\subsection{Metaphors and problematic understanding}

Based on the procedure described in Section 3, we have detected 1702 metaphors in our corpus, which we have analyzed considering their association with potential problems of understanding. The results are summarized in Table 4.

Table 4

Identification and understanding of metaphors.

\begin{tabular}{ll}
\hline How metaphors are used & Total \\
\hline Metaphors used & 1702 \\
Frequency of the metaphors in the corpus & $9 \%$ \\
Metaphors leading to problems of understanding (stronger evidence) & $110(6.4 \%)$ \\
Metaphors leading to problems of understanding (all types of evidence) & $269(15.8 \%)$ \\
Metaphors followed by an anticipation & $125(7.4 \%)$ \\
Successful metaphors (followed by a relevant reply) & $284(16.6 \%)$ \\
\hline
\end{tabular}

These figures refer only to the evidence that we can gather from written texts. We notice that the evidence can only allow us to draw some conclusions on less than $40 \%$ of the metaphors used. The remaining $60 \%$ of the metaphors are not followed by linguistic evidence of their understanding or misunderstanding.

We conducted as chi square analysis to explore the possible associations between the use of a metaphor and the detection of a misunderstanding. We detected the cases of problematic understanding according to two distinct procedures, first considering only the strong evidence (LACK, SEM ALT and PRAG ALT), and then all the types of evidence (S, A, W).

The first analysis was conducted on the problematic understanding resulting from stronger evidence. The variables considered were the nature of the move (metaphorical vs non-metaphorical) and the reply thereto expressed in the following move. The contingency table below summarizes the results (Table 5):

Table 5

Metaphors * misunderstanding crosstabulation.

\begin{tabular}{llll}
\hline Count & & Metaphor & Total \\
\cline { 2 - 4 } & & Metaphor & No metaphor \\
\hline Problematic understanding & Probl. understanding & 110 & 170 \\
& No Probl. understanding & 1592 & 16,807 \\
Total & & 1702 & 16,977 \\
\hline
\end{tabular}


We found a significant association between metaphorical moves and problematic understanding (considering only the categories providing the stronger evidence), $\chi^{2}(1)=312.51 \mathrm{p}<.001$, Phi: 0.129 , suggesting that metaphor use is positively correlated with stronger evidence of problematic understanding. Then we took into account the cases of problematic understanding resulting from all the types of evidence except for anticipation. The results of this analysis confirm the correlation, showing a slightly higher effect size $\left(\chi^{2}(1)=722.07, \mathrm{p}<.001\right.$, Phi: 0.2$)$.

To test the strength of the association between metaphors and problematic understanding, we conducted a Spearman Rank Order test having as variables the number of metaphors in the visits and the quality of understanding. This latter variable was calculated considering the number of cases of problematic understanding detected through the two distinct detection scenarios (strong evidence and all evidence). The quality of understanding was considered as an ordinal value representing the degrees of understanding (the lower the number of cases of problematic understanding, the higher the quality of understanding). In the first scenario (only strong type of evidence), we observed a strong, positive association between metaphors and problematic understanding: $r_{s}(45)=0.47 p<.001$. Similar results are found considering all the types of evidence: $\mathrm{r}_{\mathrm{s}}(45)=0.63 \mathrm{p}<.001$

\subsection{Metaphors and roles}

Exploratory statistical analysis was also performed to assess whether there was a difference between patients and healthcare providers in relation to misunderstandings resulting from the use of metaphors. An association between role (patients vs. healthcare providers) and misunderstandings caused by metaphor use (considering only the strongest type of evidence) was observed, $\chi^{2}(1)=36.111 \mathrm{p}<.001$, Phi: 0.146 . The contingency table is the following (Table 6):

Table 6

Misunderstood metaphors * role crosstabulation.

\begin{tabular}{llll}
\hline & & Role \\
\cline { 2 - 4 } & & Healthcare-provider & Patient \\
\hline Metaphor resulting in probl. understanding & Misunderstood & 40 & 1034 \\
Total & Not-Misunderstood & 10 & 1074 \\
\hline
\end{tabular}

This result shows that healthcare providers tend to use more metaphors than patients (almost the double), but patients' metaphors result in more problematic understanding than the ones used by doctors. No significant correlations were observed between the distribution of problematic metaphors and level of education (elementary vs. high school education) $\left(\chi^{2}(1)=403\right.$, n.s.).

\subsection{Metaphors and anticipation}

We assessed the relation between the use of a metaphor and the detection of an anticipation (ANT), which represents a linguistic strategy used by the speakers to rephrase a term or a sentence that they consider as potentially problematic. In other words, we assessed the awareness showed by patients and providers of the potential misunderstandings caused by their utterances (Rossi \& Macagno, forthcoming). An association between the use of a metaphor and the awareness of the risk of potential misunderstanding was observed, $\chi^{2}(1)=761.91, \mathrm{p}<.01$, Phi $=0.2$ (small effect size) (Table 7 ).

Table 7

Anticipation * metaphor crosstabulation.

\begin{tabular}{llll}
\hline Count & & Metaphor & Total \\
\cline { 3 - 4 } & & Metaphor & No metaphor \\
\hline Anticipation & Anticipation & 125 & 70 \\
& No anticipation & 1577 & 16,907 \\
Total & & 1702 & 16,977 \\
\hline
\end{tabular}

No significant difference between patient's and provider's anticipation for metaphors compared to other potential sources of problematic understanding was detected. Through anticipations, speakers rephrase a term or a sentence that include a metaphor as a linguistic strategy to clarify something they consider potentially unclear, ambiguous or vague. Anticipation constitutes an effective prevention strategy in this respect. 


\subsection{Discussion}

The aforementioned exploratory analyses, and especially the results presented in Section 6.2, provide a cue of a deeper qualitative difference in the way patients and providers use metaphors, which bring us back to the epistemic imbalance we mentioned earlier. For example, in Case 1 and Case 4 above, patients use metaphorical expressions to explain evidence they think might be relevant to improve their diabetes self-management and/or health quality. In Case 1, the patient noticed something strange about the trend of glycemia when she eats a specific type of food (gnocchi). In Case 4, the patient uses a metaphor to provide a reason why her glycemic values got worse. In these contexts, metaphorical expressions seem to be used by patients to fill lexical gaps (Goatly, 2011), namely they are instruments for sharing contents concerning symptoms or correlations between symptoms and possible causes with providers - although they are often ineffective and therefore (potentially) dangerous from a communicative point of view.

The healthcare providers' use of metaphors shows two important trends. The first aspect that is worth underscoring is that providers tend to be aware of the problems of understanding related to metaphor uses, and for this reason they tend explain the meaning of a metaphor (more than $15 \%$ of the metaphors used are explained preventively before detecting a misunderstanding). For this reason, the metaphors resulting in misunderstanding is much lower (4\% of the metaphors used are associated to problematic understanding) than the ones used by patients (11\%).

The second aspect that emerges is related to the subject matter discussed in the providers' metaphorical utterances resulting in problematic understanding. We notice that the broad topic of diabetes management (DM) represents the majority of such instances (56\%), followed by procedural issues - such as the planning of new controls or tests (14\%). The problematic metaphors related to diabetes management can be divided in two categories, namely the ones concerning the selfmeasurements (30\% of DM, $22 \%$ of the total), and the ones used to discuss aspects of the patient's lifestyle ( $70 \%$ of DM, $34 \%$ of the total). A crucial difference can be observed between these two groups. The problematic metaphors used for addressing lifestyle issues are extremely varied (they are used at most twice in the corpus) and concern a broad spectrum of issues (diet, eating habits, hypoglycemias, physical exercise, behavior on specific occasions). In contrast, even though the former sub-topic is extremely narrow (mostly consisting in explaining to the patient the frequency of the measurements and how to update the diary), the number of cases of problematic metaphorical uses is comparatively high, mostly due to some metaphors frequently recurring (such as "profile," "reasoned <measurement $>$," "reasoning," "structured $<$ measurement $>$," "where you are going < with respect to diabetes level>," "coupled <measurement $>$ ") that are not properly "technical" but that rather characterize the medical jargon.

These trends are particularly interesting for the analysis of communication between two distinct speech communities, characterized by distinct uses of the same language (Kecskes, 2008, 2013; Kecskes and Zhang, 2009). While the aforementioned "medical jargon" metaphors are used by healthcare providers with specific meanings, they need to be interpreted by patients each time, and result in reconstructions of meaning that are vague or uncertain, leading to alternative understandings. A clear example is Case 2 ("a little more reasoned manner"), in which the provider makes a recommendation relying on a quasi-technical term (used systematically by providers in this corpus), referred to a specific way of measuring the glycemia before and after a meal. As underscored in the literature on metaphor (Cameron, 2003; Gibbs, 1994; Semino, 2008), providers do not perceive these expressions as metaphorical anymore; on the contrary, due to their frequent use in medical practice, they probably and incorrectly consider them as part of the common ground that should be shared with patients. Patients are thus faced with expressions that have not a specific meaning for them, but which they can understand by reconstructing their metaphorical meaning. This reconstruction results in an expression of illusory understanding (or agreement) by the patients, which then it is often challenged by the correcting (or disagreement) moves performed by providers. From a qualitative point of view these cases are the most problematic ones as, if not resolved and repaired, they constitute a risk for the patient safety.

A final remark concerns the use of metaphors for purely "medical" explanations. The problematic metaphors used for explaining medical concepts or topics related to the disease are less than $9 \%$ of the total, followed by the ones used to refer to, describe, or assess the measurement values (8\%) and routine controls, consisting in checking skin sensitivity and the presence of possible skin conditions (7\%). As pointed out above, providers tend to be extremely careful to the patient's understanding of the general characteristics and risks of diabetes, anticipating and clarifying preventively possible misunderstandings and using different explanatory strategies at the same time (figurative language, technical language, different types of metaphors).

These figures show how the providers' awareness of their metaphor uses and the potential risks related thereto is high when the topic under discussion is technical and thus potentially results in an information or cultural gap between the interlocutors. In contrast, when the subject matter is presumed to be shared (namely concerns eating habits or checks performed by the patient), the level of awareness decreases, leading to a higher risk that the use of metaphors results in problematic understanding. A second inference can be drawn concerning the relationship between subject matter, metaphors, and problematic understanding. Considering the distribution reported above, it seems that the problems of understanding arise when the topic is very concrete and requires specific instructions (frequency, measurements, habits, quantities - of food, liquids, etc.), but the metaphors used to describe or indicate them have a vague meaning for the patient.

These results need to be considered within the limits of an exploratory work, limited to a corpus that is confined to a limited number of individuals, a specific territory, and a specific disease. Further analysis on different and broader corpora are needed both to assess the potentiality of the classification we used and to clarify when the use of a metaphor is problematic 
and why. From these empirical studies it will be possible to draw the generalizations that at present are left only as tentative conclusions.

\section{Conclusion}

The analysis of actual or potential problems of communication involves the detection of problematic understanding. More specifically, the soundness of corpus analyses depends on their replicability, which ultimately depends on the interrater agreement on the analytical criteria and the possibility of explaining, justifying, and accounting for the choices to classify a communicative event as a symptom of a misunderstanding. We described and classified the different types of evidence that can be used for detecting and justifying the problematic understanding of an utterance according to their probative weight. The types of evidence have been divided according to the varieties of problematic understanding, and the force that the evidence can provide to the conclusion that an utterance has led to an actual or potential problem of comprehension. Considering the continuum between full understanding and a complete lack of understanding, we have detected symptoms (linguistic evidence) and potential causes (metaphors) and proposed a classification of evidence of problematic understanding that can be used for bringing to light when and why the interlocutors' interpretations mismatch or may mismatch. We have applied this coding scheme to our corpus of diabetes care interviews, correlating the results with the use of metaphors.

Our analysis shows a positive correlation between evidence of misunderstandings and metaphorical utterances. In general, metaphorical utterances tend to lead to more problems of understanding than non-metaphorical ones; however, this general result needs to be assessed carefully considering the roles of the speakers and the topics under discussion. While healthcare providers use more metaphors than patients, patient's metaphors are the most problematic. Moreover, healthcare providers tend to explain their own metaphors (through anticipation moves). The problems that arise in providers' communication are mostly related to metaphors used for referring to or describing non-technical topics requiring a higher level of precision, such as habits, self-management, or dietary regime.

These results can be interpreted considering the intercultural-intracultural continuum, and in particular the distinction between the specific knowledge defining a type of interaction (medical knowledge) and the (more broadly defined) cultural dimension. The communication between providers and patients is characterized by a twofold cultural gap. The first is the "epistemic" gap that defines a medical interview, namely the different levels of technical knowledge concerning medical topics. However, this unbalance does not seem to be one of the most frequent causes of problematic understanding, as providers seem to be aware of this risk and use different communicative strategies in their medical explanations. The second difference results from different cultural communities to which providers and patients belong. As pointed out in our corpus description, providers and patients share the same linguistic code, but they have different educational levels and were born and grown in different regions of Italy. This can lead to the presumption that the common ground shared by the interlocutors is broader than it is. The prior contexts of experience (or the system or related commonplaces) associated with the meaning and the use of a linguistic expression and used for interpreting a metaphor (Macagno \& Zavatta, 2014) is thus presumed, but not actually shared by the interlocutors (Kecskes, 2013). Therefore, the evidence of problematic understanding that we found can be explained in terms of "prior contexts:" providers and patients are paying attention to different prior contexts when they use and interpret metaphors, and more importantly when they presume that a metaphor is commonly shared.

\section{Acknowledgments}

This work was supported by the Fundação para a Ciência e a Tecnologia (research grants no. PTDC/FER-FIL/28278/2017, SFRH/BPD/115073/2016, and PTDC/MHC-FIL/0521/2014).

\section{References}

Álvarez, I., Selva, L., Medina, J.L., Sáez, S., 2017. Using root metaphors to analyze communication between nurses and patients: a qualitative study. BMC Med. Educ. 17 (1), 216.

Aristotle, 1991. Poetics. In: Barnes, J. (Ed.), The Complete Works of Aristotle, vol. II. Princeton University Press, Princeton.

Arroliga, A.C., Newman, S., Longworth, D.L., Stoller, J.K., 2002. Metaphorical medicine: using metaphors to enhance communication with patients who have pulmonary disease. Ann. Intern. Med. 137 (5 part 1), 376-379.

Bigi, S., 2011. The persuasive role of ethos in doctor-patient interactions. In: Communication and Medicine, vol. 8, pp. 67-75. https://doi.org/10.1558/cam. v8i1.67.

Bigi, S., 2014a. Healthy reasoning: the role of effective argumentation for enhancing elderly patients' self-management abilities in chronic care. In: Riva, G., Ajmone Marsan, P., Grassi, C. (Eds.), Active Ageing and Healthy Living: a Human Centered Approach in Research and Innovation as Source of Quality of Life, vol. 203. IOS Press, Amsterdam, pp. 193-203. https://doi.org/10.3233/978-1-61499-425-1-193.

Bigi, S., 2014b. Key components of effective collaborative goal setting in the chronic care encounter. Commun. Med. 11 (21), 1-13. https://doi.org/10.1558/ cam.v11i2.21600.

Bigi, S., 2016. Communicating (with) Care: a Linguistic Approach to Doctor-Patient Interactions. IOS Press, Amsterdam.

Black, M., 1955. Metaphor. Proceedings of the Aristotelian Society, New Series 55, 273-294.

Black, M., 1979. More about metaphor. Metaphor and Thought 31, 19-43. https://doi.org/10.1111/j.1746-8361.1977.tb01296.x.

Bleakley, A., 2017. Thinking with Metaphors in Medicine: the State of the Art. Routledge, New York.

Cameron, L., 1999. Identifying and describing metaphor in spoken discourse data. In: Cameron, L., Low, G. (Eds.), Researching and Applying Metaphor. Cambridge University Press, Cambridge, pp. 105-132.

Cameron, L., 2002. Metaphors in the learning of science: a discourse focus. Br. Educ. Res. J. 28 (5), $673-688$. 
Cameron, L., 2003. Metaphor in Educational Discourse. Continuum, London.

Cameron, L., Maslen, R., 2010. Metaphor Analysis: Research Practice in Applied Linguistics, Social Sciences and the Humanities. Equinox, London.

Capone, A., 2009. Are explicatures cancellable? Toward a theory of the speaker's intentionality. Intercult. Pragmat. 6 (1), 55-83. https://doi.org/10.1515/ IPRG.2009.003.

Carston, R., 2009. The explicit/implicit distinction in pragmatics and the limits of explicit communication. Int. Rev. Pragmat. 1 (1), 35-62.

Casarett, D., Pickard, A., Fishman, J.M., Alexander, S.C., Arnold, R.M., Pollak, K.I., Tulsky, J.A., 2010. Can metaphors and analogies improve communication with seriously ill patients? J. Palliat. Med. 13 (3), 255-260.

Clark, H., Brennan, S., 1991. Grounding in communication. In: Resnick, L., Levine, J., Teasley, S. (Eds.), Perspectives on Socially Shared Cognition. American Psychological Association, Washington, pp. 127-149. https://doi.org/10.1037/10096-006.

Crawford, T., Candlin, S., Roger, P., 2017. New perspectives on understanding cultural diversity in nurse-patient communication. Collegian 24 (1), $63-69$.

Demjén, Z., Semino, E., 2016. Using metaphor in healthcare: physical health. In: Demjen, Z., Semino, E. (Eds.), The Routledge Handbook of Metaphor and Language. Routledge, London, pp. 385-399. https://doi.org/10.4324/9781315672953.

Demjen, Z., Semino, E., Koller, V., 2016. Metaphors for "good" and “bad” deaths: a health professional view. Metaphor Soc. World 6 (1), 1-19. https://doi.org/ 10.1075/msw.6.1.01dem.

Demmen, J., Semino, E., Demjén, Z., Koller, V., Hardie, A., Rayson, P., Payne, S., 2015. A computer-assisted study of the use of violence metaphors for cancer and end of life by patients, family carers and health professionals. Int. J. Corpus Linguist. 20 (2), 205-231.

Epstein, R., Gramling, R., 2012. What is shared in shared decision making? Complex decisions when the evidence is unclear. Med. Care Res. Rev. 70 (1), 94-112. https://doi.org/10.1177/1077558712459216.

Epstein, R., Street, R., 2011. Shared mind: communication, decision making, and autonomy in serious illness. Ann. Fam. Med. 9 (5), 454-461. https://doi.org/ $10.1370 /$ afm.1301.

Ervas, F., Gola, E., Rossi, M.G., 2015. Metaphors and emotions as framing strategies in argumentation. In: CEUR-Workshop Proceedings, vol. 1419, pp. 645-650.

Ervas, F., Montibeller, M., Rossi, M.G., Salis, P., 2016. Expertise and metaphors in health communication. Med. Stor. 16 (9-10), 91-108.

Gibbs, R., 1987. Mutual knowledge and the psychology of conversational inference. J. Pragmat. 11 (5), 561-588.

Gibbs, R., 1992. When is metaphor? The idea of understanding in theories of metaphor. Poetics Today 13 (4), 575-606.

Gibbs, R., 1994. The Poetics of Mind: Figurative Thought, Language and Understanding. Cambridge University Press, Cambridge.

Gibbs, R., Franks, H., 2002. Embodied metaphor in women's narratives about their experiences with cancer. Health Commun. 14 (2), $139-165$.

Goatly, A., 2011. The Language of Metaphors. Routledge, London.

Gumperz, J., Tannen, D., 1979. Individual and social differences in language use. In: Fillmore, C., Kempler, D., Wang, W. (Eds.), Individual Differences in Language Ability and Language Behavior. Academic Press, New York, pp. 305-325.

Jaszczolt, K., 2002. Semantics and Pragmatics: Meaning in Language and Discourse. Pearson, Harlow.

Kecskes, I., 2008. Dueling contexts: a dynamic model of meaning. J. Pragmat. 40 (3), 385-406. https://doi.org/10.1016/j.pragma.2007.12.004.

Kecskes, I., 2013. Intercultural Pragmatics. Oxford University Press, Oxford.

Kecskes, I., 2015. Intracultural communication and intercultural communication: are they different? Int. Rev. Pragmat. 7, 171-194. https://doi.org/10.1163/ $18773109-00702002$.

Kecskes, I., 2016. Can intercultural pragmatics bring some new insight into pragmatic theories? In: Mey, J., Capone, A. (Eds.), Interdisciplinary Studies in Pragmatics, Culture and Society. Springer, Cham, pp. 43-69.

Kecskes, I., Zhang, F., 2009. Activating, seeking, and creating common ground: a socio-cognitive approach. Pragmat. Cognit. 17 (2), 331-355. https://doi.org/ $10.1075 /$ pc.17.2.06kec.

Kecskes, I., Zhang, F., 2013. On the dynamic relations between common ground and presupposition. In: Capone, A., Lo Piparo, F., Carapezza, M. (Eds.), Perspectives on Linguistic Pragmatics, Perspectives in Pragmatics, Philosophy \& Psychology, vol. 2. Springer, Cham, pp. 375-395. https://doi.org/10. 1007/978-3-319-01014-4_15.

Keysar, B., Glucksberg, S., 1992. Metaphor and communication. Poetics Today 13 (4), 633-658.

Kilgarriff, A., Baisa, V., Bušta, J., Jakubíček, M., Kovár, V., Michelfeit, J., et al., 2014. The Sketch Engine: ten years on. Lexicography 1(1), 7-36.

Kovecses, Z., 2015. Where Metaphors Come from: Reconsidering Context in Metaphor. Oxford University Press, Oxford.

Kövecses, Z., 2005. Metaphor in Culture: Universality and Variation. Cambridge University Press, Cambridge.

Kövecses, Z., 2010. DELTA: Documentação de Estudos em Lingüística Teórica e Aplicada. Metaphor Lang. Cult. 26, 739-757. https://doi.org/10.1590/S010244502010000300017.

Leech, G., 1981. Semantics: the Study of Meaning, second ed. Penguin, Harmondsworth, Middlesex.

Leech, G., 1983. Principles of Pragmatics. Longman, London.

Littlemore, J., Chen, P.T., Koester, A., Barnden, J., 2011. Difficulties in metaphor comprehension faced by international students whose first language is not English. Appl. Linguist. 32 (4), 408-429.

Macagno, F., 2018a. A dialectical approach to presupposition. Intercult. Pragmat. 15 (2), 291-313. https://doi.org/10.1515/ip-2018-0008.

Macagno, F., 2018b. Evidence and presumptions for analyzing and detecting misunderstandings. Pragmat. Cognit. 24 (2), 263-296.

Macagno, F., 2018c. Assessing relevance. Lingua 210-211, 42-64. https://doi.org/10.1016/j.lingua.2018.04.007.

Macagno, F., Capone, A., 2016. Interpretative disputes, explicatures, and argumentative reasoning. Argumentation 30 (4), 399-422. https://doi.org/10.1007/ s10503-015-9347-5.

Macagno, F., Zavatta, B., 2014. Reconstructing metaphorical meaning. Argumentation 28 (4), 453-488. https://doi.org/10.1007/s10503-014-9329-Z.

Mauranen, A., 2006. Signaling and preventing misunderstanding in English as lingua franca communication. Int. J. Sociol. Lang. 177, 123-150. https://doi. org/10.1515/IJSL.2006.008.

Musolff, A., 2014. Metaphors: sources for intercultural misunderstanding? Int. J. Lang. Cult. 1 (1), 42-59.

Musolff, A., 2015. Metaphor interpretation and cultural linguistics. Lang. Semiot. Stud. 1 (3), 35-51.

Naik, A.D., Teal, C.R., Rodriguez, E., Haidet, P., 2011. Knowing the ABCs: a comparative effectiveness study of two methods of diabetes education. Patient Educ. Counsel. 85 (3), 383-389. https://doi.org/10.1016/j.pec.2011.01.010.

Rossi, M.G., 2016. Metaphors for patient education. A pragmatic-argumentative approach applying to the case of diabetes care. Rivista Italiana Di Filosofia Del Linguaggio $10(2), 34-48$.

Rossi, M.G., Leone, D., Bigi, S., 2017. The ethical convenience of non-neutrality in medical encounters: argumentative instruments for healthcare providers. Teoria 37 (2), 139-157.

Rossi, M.G., Macagno, F., 2019. Coding problematic understanding within patient-provider interactions. Forthcoming.

Sacks, H., Jefferson, E., Schegloff, G., 1974. A simplest systematics for the organization of turn-taking for conversation. Language 50 (4), $696-735$.

Schegloff, E., 1987. Some sources of understanding in talk-in-interaction. Linguistics 25, 201-218. https://doi.org/10.1515/ling.1987.25.1.201.

Schegloff, E., Jefferson, G., Sacks, H., 1977. The preference for self-correction in the organization of repair in conversation. Language 53 (2), 361-382. https:// doi.org/10.2307/413107.

Schiappa, E., 2003. Defining Reality. Definitions and the Politics of Meaning. Southern Illinois University Press, Carbondale and Edwardsville.

Semino, E., 2008. Metaphor in Discourse. Cambridge University Press, Cambridge.

Semino, E., Demjén, Z., Demmen, J., 2016. An integrated approach to metaphor and framing in cognition, discourse, and practice, with an application to metaphors for cancer. Appl. Linguist. 39 (5), 625-645.

Semino, E., Demjén, Z., Demmen, J., Koller, V., Payne, S., Hardie, A., Rayson, P., 2015. The online use of violence and journey metaphors by patients with cancer, as compared with health professionals: a mixed methods study. BMJ Support. Palliat. Care 7 (1), 60-66. 
Semino, E., Demjen, Z., Hardie, A., Payne, S.A., Rayson, P.E., 2018. Metaphor, Cancer and the End of Life: A Corpus-Based Study. Routledge, London.

Semino, E., Heywood, J., Short, M., 2004. Methodological problems in the analysis of metaphors in a corpus of conversations about cancer. J. Pragmat. 36 (7), $1271-1294$.

Sharifian, F., 2014. Conceptual metaphor in intercultural communication between speakers of Aboriginal English and Australian English. In: Musolff, A., MacArthur, F., Pagani, G. (Eds.), Metaphor and Intercultural Communication. Bloomsbury, London, pp. 117-129.

Sperber, D., Wilson, D., 1995. Relevance: Communication and Cognition. Blackwell Publishing Ltd, Oxford.

Steen, G., 2010. When is metaphor deliberate? In: Alm-Arvius, C., Johannesson, N.-L., Minugh, D. (Eds.), Selected Papers from the Stockholm 2008 Metaphor Festival (Stockholm Studies in English). University of Stockholm, Stockholm, pp. 43-63.

Steen, G., Dorst, A., Herrmann, B., Kaal, A., Krennmayr, T., Pasma, T., 2010. A Method for Linguistic Metaphor Identification: from MIP to MIPVU. John Benjamins Publishing Company, Amsterdam.

Street Jr., R., Makoul, G., Arora, N., Epstein, R., 2009. How does communication heal? Pathways linking clinician-patient communication to health outcomes. Patient Educ. Counsel. 74 (3), 295-301.

Trogen, B., 2017. The evidence-based metaphor. JAMA 317 (14), 1411-1412. https://doi.org/10.1001/jama.2016.17219.

Tzanne, A., 2000. Talking at Cross-Purposes: the Dynamics of Miscommunication. John Benjamins Publishing, Amsterdam.

Wagner, E., Austin, B., Davis, C., Hindmarsh, M., Schaefer, J., Bonomi, A., 2001. Improving chronic illness care: translating evidence into action. Health Aff. 20 (6), 64-78. https://doi.org/10.1377/hlthaff.20.6.64.

Walton, D., 2016. Argument Evaluation and Evidence. Springer, Cham.

Walton, D., Reed, C., 2008. Evaluating corroborative evidence. Argumentation 22 (4), 531-553. https://doi.org/10.1007/s10503-008-9104-0.

Wikberg, K., 2004. English metaphors and their translation. In: Aijmer, K., Stenström, A.-B. (Eds.), Discourse Patterns in Spoken and Written Corpora. John Benjamins, Philadelphia, pp. 245-265.

Yus, F., 1999. Misunderstandings and explicit/implicit communication. Pragmatics 9 (4), 487-517. https://doi.org/10.1075/prag.9.4.01yus.

Fabrizio Macagno (Ph.D. UCSC, Milan, 2008) works as a researcher and invited auxiliary professor at the Universidade Nova de Lisboa. He is author of more than 80 papers on definition, presupposition, argumentation schemes, and dialogue analysis published on major international peer-reviewed journals such as Journal of Pragmatics, Intercultural Pragmatics, Argumentation, and Philosophy and Rhetoric. His most important publications include the books Argumentation Schemes (CUP 2008), Emotive language in argumentation (CUP 2014), and Interpreting straw man argumentation (Springer 2017).

Maria Grazia Rossi (Ph.D Università di Messina, Messina, 2012) works as post-doc researcher at the Universidade Nova de Lisboa. Her current research, in the fields of philosophy of language and healthcare communication, focus on the role of metaphors as argumentative tools to foster patient understanding. She has worked on metaphors and emotions as reasoning and decision-making tools, especially in the medical and moral context. She published a monograph, several articles and edited books, among which "Metaphor in Communication, Science and Education" (De Gruyter, 2017, with F. Ervas and E. Gola), "The ethical convenience of non-neutrality in medical encounters. Argumentative instruments for healthcare providers" (Teoria, 2017, Rossi M.G., Leone D. \& Bigi S.) and "Metaphors for patient education: a pragmatic-argumentative approach applying to the case of diabetes care" (RIFL, 2016). 